\title{
LA TRAZA CON QUE «HARÁ LA MÚSICA MILAGROS»: UN TONO DEL MAESTRO CAPITÁN PARA UNAS DÉCIMAS DE LOPE DE VEGA
}

Resumen: En esta comunicación se intenta demostrar, con un ejemplo concreto, cómo el compositor Mateo Romero, llamado el Maestro Capitán, se inspira en el inicio melódico de una canción tradicional para componer un tono humano de calidad artística incuestionable, teniendo en cuenta, además, que el texto poético de ese tono es de Lope de Vega, quien, por su parte, glosó el primer verso de esa tonada tradicional en unas décimas. Es decir, que músico y poeta bebieron de fuentes populares, y ambos glosaron, comentaron y recrearon el incipit tradicional. El trabajo hace referencia también al recopilador de la canción tradicional, Francisco Salinas, y a Vicente Espinel, creador de la estrofa denominada décima espinela. El circuito artístico generado demuestra la espléndida intertextualidad poético-musical que subyace en esa composición, la cual puede erigirse en paradigma de una manera de componer característica del siglo XVII.

\section{THE TRAZA OF «HARÁ LA MÚSICA MILAGROS»: A TUNE FROM MAESTRO CAPITÁN FOR LOPE DE VEGA'S DÉCIMAS}

Abstract: This dissertation has the intention to demonstrate, with a concrete example, how the composer Mateo Romero, also called Maestro Captain, inspired by the melodic beginning of a traditional song, composed a human tune of unquestionable artistic quality, considering, in addition, that the poetic text for that tune belongs to Lope de Vega, who had already glossed the first verse of that traditional song on a few décimas. This means that musician and poet drank from popular sources and both of them glossed, commented and recreated the traditional incipit. The work also refers to the traditional song's compiler Francisco Salinas, and to Vicente Espinel, creator of the verse called décima espinela. The created artistic circuit shows the magnificent musical-poetic intertextualiy underlying that 
composition, which can become a paradigm of a typical way of composing in XVII century.

Refiriéndonos a la música profana y a las letras a lo humano de los siglos XVI y XVII, resulta ya un tópico recurrente y falto de novedad afirmar que en nuestra Edad de Oro la música y la poesía formaban una unidad expresiva indisoluble, en la que ambas artes se interrelacionaban e influenciaban mutuamente. Lo que ya no es tan recurrente ni falto de novedad e interés es que, además, conforman a nuestra mirada un sutil y complejo circuito artístico cuya cabal aprehensión y análisis son tan enriquecedores para los investigadores, musicólogos y filólogos, como atractivos y sugerentes para los intérpretes de nuestra música antigua.

Es nuestra intención centrarnos en este circuito artístico generado a partir de una composición poético-musical que se nos ha transmitido en el Cancionero de la Sablonara y que se titula «¿A quién contaré mis quejas?», en cuya realización convergen de manera directa o indirecta los siguientes autores que, con sus obras y referencias, la hacen posible, al tiempo que se erigen en un magnífico ejemplo del método creativo en la Edad de Oro: la imitación compuesta, con el que la originalidad estriba en dejar al descubierto las huellas creativas de maestros u obras anteriores. Es decir, un método con el que la tradición se asienta como fundamento obligado de todo acto creador. Citamos esos autores en el orden que seguiremos en nuestra argumentación.

En primer lugar, el teórico musical Francisco Salinas (1513-1590) con su tratado De musica libri septem $(1577)^{1}$. Después el Fénix de los ingenios, Lope de Vega (1562-1635), que escribió la novela pastoril Arcadia (1598) ${ }^{2}$ y el famoso poema preceptista Arte nuevo de hacer comedias en este tiempo $(1609)^{3}$. Seguiremos después con el compositor Mateo Romero, llamado

1. Palisca, Claude V. «Salinas, Francisco de». En: Diccionario de la Música Española e Hispanoamericana. (Director Emilio CASARES RODICIO). Madrid: Sociedad General de Autores y Editores, 2002, vol. 9, pp. 598-602. SALINAS, Francisco. De musica libri septem. (Edición facsímil de Macario Santiago KASTNER). Kassel und Basel: Bärenreiter Verlag, 1958. SALINAS, Francisco. Siete libros sobre la música. (Primera versión castellana por Ismael FERNÁNDEZ DE LA CUESTA). Madrid: Editorial Alpuerto, 1983.

2. Lope DE VegA. Arcadia. (Edición, introducción y notas de Edwin S. Morby). Madrid: Editorial Castalia, 1975.

3. LOPE DE VEGA. El arte nuevo de hacer comedias en este tiempo. (Edición y estudio preliminar de Juana de JosÉ PRADES). Madrid: Consejo Superior de Investigaciones Científicas, 1971. 
el «Maestro Capitán» (ca. 1575-1647), músico inspirado que trabajó brillantemente en las cortes de Felipe III y Felipe IV ${ }^{4}$. Y terminaremos con el humanista, poeta y músico Vicente Espinel (1550-1624) a cuya novela Relaciones de la vida del escudero Marcos de Obregón (1618) será preciso acu$\operatorname{dir}^{5}$.

Salinas, desde su formación y espíritu humanista, incluyó en su tratado anteriormente citado y con la intención de «ejemplificar, con cánticos hispánicos, el tipo métrico» ${ }^{6}$, casi un centenar de referencias tradicionales, entre las que figuran melodías más o menos completas, fragmentos de melodías, pequeños incisos melódicos o citas poéticas sin música ${ }^{7}$. Algunas de esas melodías ya habían merecido la atención de músicos y compositores con anterioridad a la edición del libro de Salinas. Otras, posteriores, habían sido recreadas o arregladas en versiones polifónicas o

4. BeCQuART, Paul. «Romero, Mateo. El Maestro Capitán». En: Diccionario de la Música..., vol. 9, pp. 381-383.

5. Espinel, Vicente. Vida del escudero Marcos de Obregón. (Edición, introducción y notas de Ma Soledad CARrASCO URGOITI). Madrid: Editorial Castalia, 1972, 2 vols.

6. Vid. Alín, José Ma. «Francisco Salinas y la canción popular del siglo XVI». En: Lírica popular. Lírica tradicional. Lecciones en homenaje a Don Emilio García Gómez. (Edición de Pedro M. PIÑ̃ERO RAMírEZ). Sevilla: Universidad de Sevilla. Fundación Machado, 1998, p. 144. Vid. también Otaola, Paloma. El humanismo musical en Francisco Salinas. Pamplona: Newbook Ediciones, 1997.

7. Ismael Fernández de la Cuesta, en su edición española del tratado de Salinas ya citada, comenta que «los aportes de Salinas al conocimiento del folklore español de la época ya los había señalado F. Pedrell. Desgraciadamente, los ejemplos que trae son, en su mayoría, incompletos. Así y todo, su valor documental es muy grande» (p. 17). Por otra parte, algunas de las melodías recogidas por Salinas han llegado prácticamente hasta nuestros días, como ha demostrado $\mathrm{M}$. GARCÍA MATOS en su artículo «Pervivencia en la tradición actual de canciones populares recogidas en el siglo XVI por Salinas en su tratado De musica libri septem». En: Anuario Musical, XVIII (1963), pp. 67-84. Por su interés citamos el siguiente párrafo: «Fenómeno muy destacado es el de la desaparición y sustitución por otros, en las actuales canciones, de los textos literarios que en las de Salinas figuran. Como se habrá reparado, ni siquiera surge un solo caso de aquéllas en que, junto a la melodía, se haya también conservado el viejo cantar poético. Semejante fenómeno dase con canciones de más recientes épocas pasadas y hasta de la moderna. Su significado es claro, y puede enunciarse diciendo que la música popular tiene una capacidad de persistencia mayor que la poesía de igual género. Infinitamente mayor, cabe aún afirmar apoyándonos en multitud de otros datos e indicios que la experiencia nos suministra. Probable es que la causa principal de ello estribe en la ambigüedad o impreciso sentido de la materia musical, cualidad que, sobre conferir a ésta el etéreo carácter y poder de seducción que todos sabemos y que facilita su perennidad, o, a lo menos, su larga duración, hace posible que se le puedan adscribir o acoplar, en cualquier tiempo y sin modificarla profundamente, los textos poéticos más varios de expresión y contenido. Sucede cosa muy distinta con la poesía o canción literaria. Por lo concreto y explícito de la palabra, su materia, cansa y se consume antes en el uso, desvaneciéndose, en consecuencia, antes, también, que la música o músicas a que se aplica y siendo reemplazada por otras» (p. 81; la cursiva es del autor). 
instrumentales. Podemos constatar, en definitiva, que una treintena aproximada de esas melodías mereció la atención de los compositores con fines de cita, reutilización o dignificación. Entre ellas se halla la célebre «canción que se llama Las quexas» (Ejemplo 1), que Salinas incluyó en el libro VI de su tratado, dedicado a la rítmica ${ }^{8}$, y que Margit Frenk recoge también en su corpus, relacionando las fuentes correspondientes ${ }^{9}$ :

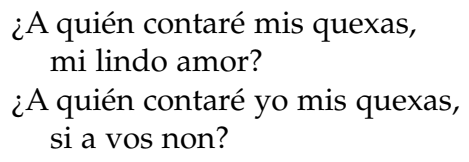

La tonada popular tiene un ámbito de una octava y presenta unos giros melódicos de evidente sabor tradicional. Es muy significativo, por ejemplo, el intervalo de tercera menor que se da al final de la primera parte de la melodía. También es significativa su disposición modal: la primera parte puede estar escrita en el modo o tono de Mi, pero la segunda parte tiene el final en Do. También podemos concluir diciendo que la canción presenta una oscilación entre los modos IV y V, o que está escrita en el tono o modo de Do, con un ámbito que va desde el Do2 al Do3 y que no trae alteraciones en la armadura.

Recurramos ahora al Fénix. Lope de Vega dejó escrito, en su poema preceptista Arte nuevo de hacer comedias en este tiempo (1609), que «las décimas son buenas para quejas» ${ }^{10}$. Con anterioridad, en la novela pastoril Arcadia (1598), el poeta ya había puesto en práctica la observancia contenida en ese endecasílabo y había incluido cinco décimas en boca del pastor Olimpio. Copio el párrafo que nos interesa, y a continuación las décimas ${ }^{11}$ :

Desesperóse Olimpio de ver favorecido a Menalca con una flor que de su guirnalda le había dado Isbella; y así en todo el camino no habló palabra, sino, mirándola a hurto de los otros pastores, daba de cuando en cuando unos mudos sospiros en que sin lengua reprehendía su ingratitud y mudanza; que antes que la pastora hubiese visto a Menalca dicen que agradecía la voluntad de Olimpio; pero cuando la mujer aborrece lo que algún tiempo le agradó, es mucho peor que si

8. Vid. Salinas. De musica..., p. 326. SAlinas. Siete libros..., p. 568.

9. Vid. FRENK, Margit. Nuevo corpus de la antigua lírica popular hispánica (siglos XV a XVII). México: Universidad Nacional Autónoma de México. El Colegio de México. Fondo de Cultura Económica, 2003, vol. I, pp. 285-289.

10. Lope DE VeGA. El arte nuevo..., p. 297. Para más información sobre este endecasílabo, vid. las pp. 193-197.

11. Lope DE Vega. Arcadia..., pp. 185-187. 
siempre lo hubiese aborrecido. Al fin, persuadido Olimpio de la fuerza de su mal, quiso darle a entender cantando así:

[1.] ¿A quién contaré mis quejas, cuando de oíllas te guardes, pues que ya tengo cobardes piedras, paredes y rejas? Y ¿adónde iré si me dejas, siendo el alma que me anima? Vuelve, señora, y estima el mal con que me atormentas; que es lástima que no sientas lo que a las piedras lastima.

[2.] Si el largo tiempo no fuerza mis agravios y tus daños, en la mitad de mis años habré de morir por fuerza; que si la vida se esfuerza con una flaca esperanza, vana fue la confianza de pensar que una mujer, en dejando de querer, deje de tomar venganza.

[3.] Porque de varios caminos has hecho prueba en mi fe; que quien sin pasión los ve, dice que son desatinos. Vuelve tus ojos divinos a mis lágrimas humanos, que vengarse es de tiranos; baste que para mi mengua remita el tiempo a mi lengua los agravios de tus manos.

[4.] Yo me acuerdo, hermosa Isbella, y estas selvas son testigos, que juramos ser amigos junto aquesta fuente bella; y que mirándote en ella, por más señas, te di aviso del loco amor de Narciso; mas ¿qué mayor que querer persuadir una mujer que aborrece lo que quiso? 
[5.] De este mi penar se arguye, según le tengo por fuerte, que aun hasta la propia muerte de los desdichados huye; el alma me restituye, si la estimas en tan poco; pero en vano te provoco, que, puesto que me la des, no querrá vivir después en aposento de loco.

Como bien señala Morby, estas décimas «vienen a ser una especie de glosa de un verso inicial antiguo y ya popular en días de Lope» ${ }^{12}$. O sea, que lo que el Fénix hizo fue recurrir al acervo popular, al inicio de un cantarcillo conocido por todos, para glosarlo líricamente e insertarlo en un marco pastoril como es la Arcadia.

Tratemos ahora del comportamiento de la música. En primer lugar cabe preguntarse qué melodía cantaría Olimpio en la ficción literaria, o mejor dicho, qué música vendría, quizá de manera inconsciente, a la mente de Lope cuando hizo que el pastor cantara sus quejas. Sin duda, la que conocían todos, la que Salinas fijó en su tratado como canción quejosa por antonomasia, aquella que el propio tratadista calificaba de «tan conocida música.» La cronología en base a fuentes documentales nos apoya: el teórico musical publicó su De musica libri septem en 1577 y el poeta su Arcadia en 1598.

Décadas más tarde, el compositor y maestro de capilla Mateo Romero puso música a las décimas de Lope, la misma música para cada décima, pero no utilizó las cinco, sino sólo las tres primeras, y no en el mismo orden en el que están en la Arcadia, lo cual viene a demostrar que el compositor ideó una traza para componer, y en esa traza entra la manipulación de la poesía: en este caso, la supresión de estrofas y el cambio de su orden. En otros casos que hemos visto, el propio compositor, o un poeta que trabaje para él, crea otras estrofas, introduce variantes, incluye un estribillo; en definitiva, poeta y músico recurren a unas trazas para componer que ahora no podemos referir en aras de la brevedad ${ }^{13}$. Volviendo a la pieza que nos ocupa, el maestro Capitán hizo servir idéntica estrate-

12. Ibidem, p. 185, n. 112.

13. Vid. nuestro trabajo: JosA, Lola y LAMBEA, Mariano. «Las trazas poético-musicales en el romancero lírico español». En: Edad de Oro, XXII (2003), pp. 29-78. 
gia compositiva que el Fénix, es decir, recurrió al venero popular. Tomó, pues, de la música tradicional, su arranque melódico, para después glosarlo armónica y contrapuntísticamente, e incluir la pieza completa a dos voces en un cancionero poético-musical de repertorio cortesano perteneciente al primer cuarto del siglo XVII, como es el Cancionero de la Sablona$r a^{14}$ (véase la música del tiple en el Ejemplo 2) ${ }^{15}$. Mateo Romero citó las cuatro primeras notas de la melodía recogida por Salinas; ciertamente un motivo musical breve, pero suficiente para la referencia musical intertextual, para el reconocimiento de la tonada popular por parte del auditorio de la época. Las ligeras diferencias rítmicas entre ambas melodías son más visuales que efectivas.

Al término ya de nuestro circuito artístico hemos de citar al cuarto personaje que nos falta: Vicente Espinel ${ }^{16}$. Y lo citaremos por dos razones: en primer lugar porque a él debemos la invención de la décima, llamada también espinela. El poeta "presentó esta estrofa en varias de las composiciones de su libro Diversas rimas $(1591)^{17}$.» Más tarde el propio Lope, en su Laurel de Apolo (1630), otorgaría autoría definitiva a Espinel con estos endecasílabos: «pues de Espinel es justo que se llamen/ y que su nombre eternamente aclamen ${ }^{18} . » Y$ en segundo lugar porque en sus Relaciones de la vida del escudero Marcos de Obregón, novela en la que se mezclan el relato picaresco, las aventuras y el fondo autobiográfico, Espinel escribe un párrafo que expone buena parte de los matices expresivos y de los conceptos que más nos gustan leer y tener a mano a los musicólogos y filólogos que, desde la perspectiva del trabajo interdisciplinario, sumamos esfuerzos para estudiar, analizar y entender las rela-

14. Vid. la edición moderna de esta importante colección del primer cuarto del siglo xVII: El Cancionero de la Sablonara. (Edición crítica, introducción y notas por Judith ETZION). London: Tamesis Books, 1996.

15. El manuscrito original del Cancionero de Claudio de la Sablonara se halla en la Bayerische Staatsbibliothek (Munich). Existe una copia fiel de este manuscrito (s. XIX) conservada en la Biblioteca Nacional (Madrid), que es la que hacemos servir para nuestra ejemplificación.

16. Griffiths, John. «Martínez Espinel, Vicente». En: Diccionario de la Música..., 2000, vol. 7, pp. 286-288.

17. Vid. NAVArro Tomás, T. Métrica española. Barcelona: Editorial Labor, 1991, p. 268.

18. Ibidem, p. 268. En otras obras cita Lope a Espinel como autor de la décima espinela; por ejemplo, en La Dorotea (1632): «A peso de oro habíades vos de comprar un hombrón de hecho y de pelo en pecho, que la desapasionase destos sonetos, y destas nuevas décimas o espinelas que se usan; perdóneselo Dios a Vicente Espinel que nos trujo esta novedad y las cinco cuerdas de la guitarra con que ya se van olvidando los instrumentos nobles.» Vid. LOPE DE VEGA. La Dorotea. (Edición de José Manuel BlECUA). Madrid: Ediciones Cátedra, 1996, acto I, escena VII, p. 154. 
ciones entre la música y la poesía en la Edad de Oro. Éste es el párrafo en cuestión ${ }^{19}$ :

[...] un día, acabando de cantar y tañer y quedando todos suspensos, preguntó uno que cómo la música no hacía ahora el mismo efecto que solía hacer antiguamente, suspendiendo los ánimos y convirtiéndolos a transformarse en los mismos conceptos que iban cantando [...]. Dije yo a esto: «Lo mismo se puede hacer ahora, y se hace.» Replicóme diciendo que después que se perdió el género enharmónico no se podía hacer. Dije yo: «Con el género enharmónico me parece que era imposible hacerse, porque como la excelencia de ese género consiste en la división de semitonos y dieses, no puede la voz humana obedecer a tantos semitonos y dieses como aquel género tiene [...]. Y que se pueda hacer y se hace con el género diatónico y cromático, como haya las mismas circunstancias y requisitos que el caso quiere, sucederá cada día lo mismo; y en las sonadas españolas, que tan divino aire y novedad tienen, se ve cada día ese milagro [se refiere a la suspensión de los ánimos]. Los requisitos son: [lo primero] que la letra tenga conceptos excelentes y muy agudos, con el lenguaje de la misma casta; lo segundo, que la música sea tan hija de los mismos conceptos que los vaya desentrañando; lo tercero es que quien lo canta tenga espíritu y disposición, aire y gallardía para ejecutarlo; lo cuarto, que el que lo oye tenga el ánimo y gusto dispuesto para aquella materia. Que desta manera hará la música milagros.»

\section{A guisa de epílogo}

Hemos cerrado nuestro circuito artístico, interrelacionando a los cuatro protagonistas que lo han conformado: Salinas, Lope, Romero y Espi-

19. Espinel. Vida del escudero..., vol. I, Relación III, Descanso V, p. 146. También cita Espinel a Francisco Salinas: «Vi al abad Salinas, el ciego, el más docto varón en música especulativa que ha conocido la antigüedad» (ibidem, p. 198). Para nuestro interés hacemos constar que Vicente Espinel utilizaba la expresión «música de sala» para referirse a la música de cámara, y que, en amplio sentido, debe entenderse aquí tanto la música vocal como la instrumental: «que la música instrumental de sala, tanto más tiene de dulzura y suavidad, cuanto menos de vocería y ruido» (ibidem, p. 100). Por otra parte Andrés PARDO TOVAR, en su artículo «Perfil y semblanza de Vicente Espinel». En: Revista Musical Chilena, 79 (1962), p. 14, recoge en nota a pie de página la siguiente información: «En las adiciones con que el Dr. Cristóbal Suárez de Figueroa enriqueció su traducción de la Plaza Universal de todas las profesiones del mundo [sic. Madrid, 1615], obra enciclopédica de Tommaso Garzoni (1549-1589), se atribuye a Espinel la invención de un nuevo género musical: las Sonadas y cantar de sala». Cf. GARCíA FraILE, Dámaso. «Música y literatura a principios del siglo XVII: Vicente Espinel (1550-1624)». En: Música y literatura en la Península Ibérica: 1600-1750. Actas del Congreso Internacional (Valladolid, 20-21 de febrero de 1995). (Edición a cargo de María Antonia Virgili Blanquet, Germán Vega García-Luengos y Carmelo CaballeRO FERNÁNDEZ-RUfETE). Valladolid: V Centenario Tratado de Tordesillas, 1998, pp. 333-346. 
nel, representantes respectivos de la teoría musical, de la poesía, de la música y de la preceptiva literaria; y hemos podido apreciar cómo, en efecto, ha hecho «la música milagros». En lo que se refiere a nuestra trayectoria científica, no nos cansaremos de repetir que el romancero lírico hispánico es un solo arte poético-musical, es decir, un arte que requiere del esfuerzo unido de filólogos y musicólogos. Por lo tanto, desde la ciencia, tenemos la obligación de reconstruir y estudiar lo que una concepción artística tan integral (interdisciplinaria, diríamos hoy) como la barroca ofrecía en perfecta simbiosis al oyente del siglo XVII. Evitemos, así pues, que la especialización mal entendida y otros aislamientos de nuestros días se interpongan y desvirtúen esa espléndida unión de dos artes que, al hermanarse, alcanzaron la máxima representación artística en uno de los movimientos estéticos más representativos de la cultura hispánica.

\section{Ejemplo 1}

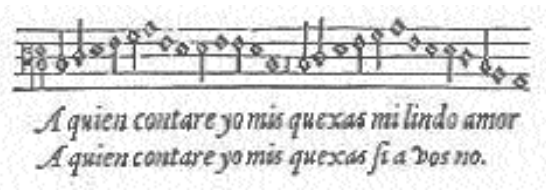

Francisco Salinas: De musica, p. 326

Francisco Salinas: Siete libros sobre la música, p. 568

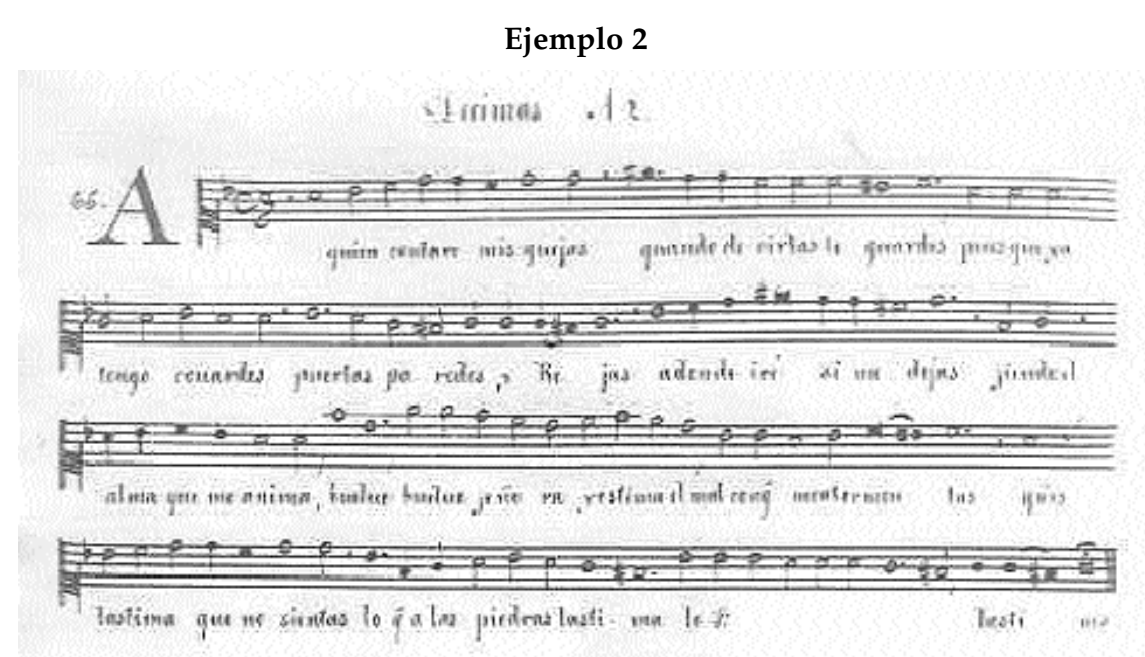

Madrid, Biblioteca Nacional, M. 1263, f. 68v 